\title{
The centrosomal localization of KM-HN-1 (MGC33607) depends on the leucine zipper motif and the $\mathrm{C}$-terminal coiled-coil domain
}

\author{
Hye Jeong Park, Hyun-Joo Seo ${ }^{1}$, Hyun-Woo Kim', \\ Jung Soon $\mathrm{Kim}^{1}$, So-Yoon Hwang ${ }^{1}$ and \\ Yeon-Sun Seong ${ }^{1,2}$

\section{${ }^{1}$ Department of Biochemistry} \\ College of Medicine, Dankook University \\ Cheonan 330-714, Korea \\ ${ }^{2}$ Corresponding author: Tel, 82-41-550-3875; \\ Fax, 82-41-550-3905; E-mail, seongys@dku.edu
}

Accepted 23 November 2007

Abbreviations: $\gamma$-TuRC, gamma-tubulin ring complex; NES, nuclear export signal

\begin{abstract}
KM-HN-1 is a C-terminal coiled-coil domain containing protein previously referred to as image clone MGC33607. This protein has been previously identified as a cancer/testis antigen and reported as nuclear and chromatin localizing protein. We raised polyclonal antisera with the GST fusion protein and identified them as a $105 \mathrm{kDa}$ protein. Motif analysis showed that this protein harbors the leucine zipper motif in internal $1 / 3$ region and the coiled-coil domain in the C-terminal region. Using the full length and various deletion mutants, we determined the motif that governs the subcellular localization of KM-HN-1. Immunofluorescence staining of the endogenous $\mathrm{KM}-\mathrm{HN}-1$ and various kinds of GFP-tagged KM-HN-1 revealed that KM-HN-1 localizes to the centrosomes as well as nucleus. The centrosomal localization-determining region of this protein is C-terminal coiled-coil domain in which the leucine zipper motif and the nuclear export signal (NES) harbor.
\end{abstract}

Keywords: CCDC110 protein, human; cell nucleus; centrosome; protein structure, tertiary; protein transport

\section{Introduction}

KM-HN-1 (MGC33607) was identified as a novel human cancer/testis antigen, in an attempts to discover cancer-specific target genes for cancer therapeutics development. From the RT-PCR analysis of cancer tissues and cancer cell lines, the mRNA expression level of $\mathrm{KM}-\mathrm{HN}-1$ was shown to be increased in a variety of cancer types, including tongue cancer, melanoma, hepatocellullar carcinoma (Monji et al., 2004; Condomines et al., 2007). We have obtained a KM-HN-1 clone from a yeast two-hybrid screening, using hCdc14A as bait. hCdc14 is one of the human orthologues of budding yeast cdc14p. Cdc14 is a dual-specificity protein phosphatase, which counteracts the cdc2/ cyclin B1. Cdc14 plays a key role in the mitotic exit network and the centrosome cycle, from budding yeasts to metazoans (Pereira and Schiebel, 2001; Trautmann and McCollum, 2002; Stegmeier and Amon, 2004). hCdc14A also performs a crucial function in centrosome duplication and the completion of mitosis in human cells (Kaiser et al., 2002; Mailand et al., 2002). The KM-HN-1 protein was recognized by cellular and humoral immune responses from a number of cancer patients suffering from a variety of cancer types. KM-HN-1 has another splicing variant in which 80-116 amino acids of $\mathrm{KM}-\mathrm{HN}-1$ are missing (Figure 1). KM-HN-1 harbors a C-terminal coiled-coil domain in which the C-terminal half of the SMC (structure maintenance of chromatin) motif resides. The SMC motif originates from SMC protein, which performs pivotal function during chromosome segregation (Cobbe and Heck, 2000). In addition to the coiled-coil domain $\mathrm{KM}-\mathrm{HN}-1$ harbors a bipartite NLS (nuclear localization signal) (Boulikas, 1993) followed by a leucine zipper region, in the internal $1 / 3$ region (Figure 1 ). The leucine zipper motif is generally comprised of five repeats of leucine ( $L 1$, L2, L3, L4, L5) in seven amino acid intervals, and this motif is preceded by basic amino acids. The leucine zipper motif functions as a protein dimerization domain of a variety of DNA binding proteins (Landschulz et al., 1988). We identified $\mathrm{KM}-\mathrm{HN}-1$ as a $105 \mathrm{kDa}$ protein via immunoblotting followed by immunoprecipitation. The clones initially obtained evidenced centrosomal localization and colocalization with hCdc14A in the centrosome. However Monji et al. (2004) previously reported that $\mathrm{KM}-\mathrm{HN}-1$ is a nuclear protein which evidences colocalization with chromatin. In this study, we found that $\mathrm{KM}-\mathrm{HN}-1$ localize to centrosomes as 
A

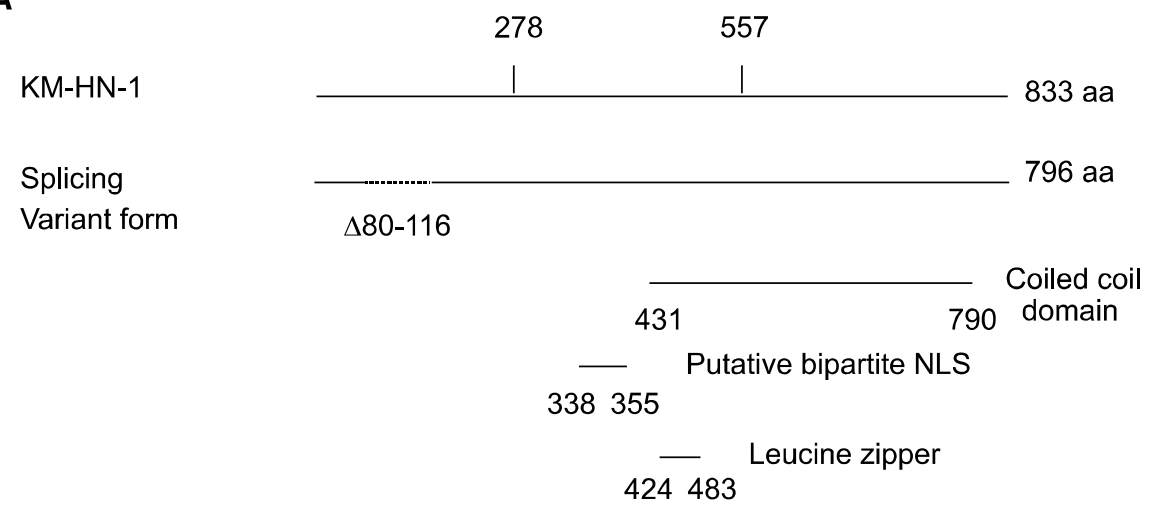

B

KM-HN-1
Jun
Fos
hc-Myc
hC/EBP
GCN4
CREB

KM-HN-1
Jun
Fos
hc-Myc
hC/EBP
GCN4
CREB

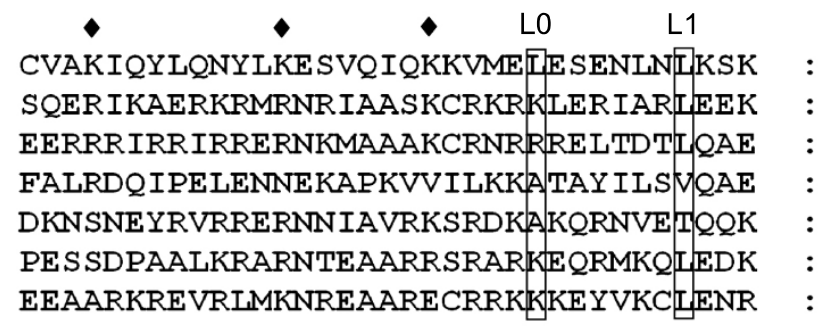

L2 L3 L4 L5

MKPIIFTTQSUIQKVETYYKQLKNUVE-------- : VKTLKAQNSEL TASTANMI TEQVAOLKO------- : TDQIEDKKSAI QTE IANL LKEKEKITF------- : EQKII ISEEDLILRKRREOIKHKLEOIRN------- : VLELTSDNDRIRKRVEQI SRELDTIRG-------- : VEELLSKNYHIENEVARI KKLVGER---------- : VAVLENONKTI IEELKAIKDLYCHKSD :
Figure 1. Schematic illustrations of KM$\mathrm{HN}-1$ and its splice variant and conserved motifs. (A) KM-HN-1 consists of 833 amino acid residues; the splicing variant $(796$ amino acid residues) has missing 80-116 of $\mathrm{KM}-\mathrm{HN}-1$. Both harbor a C-terminal coiled- coil region in which the SMC motif resides. The internal $1 / 3$ region harbors leucine zipper (LZ) region preceded by a putative bipartite NLS. (B) Amino acid alignment of leucine zipper motif. KM$\mathrm{HN}-1$ has at least five leucine zipper repeats. Key leucine zipper domain molecule (L0-L5) region of KM-HN-1 and their aligned replacements are shaded. The closed diamond $(\bullet)$ represents conserved basic amino acid residues. well as nucleus. We also determined the region of KM-HN-1 critical to centrosomal localization, and other features of the intracellular localizations using $\mathrm{KM}-\mathrm{HN}-1$ deletion mutants.

\section{Materials and Methods}

\section{Cell culture}

A U-2 OS human osteosarcoma cell line (American Type Culture Collection HTB 96) and a 293 human embryonic kidney cell line (American Type Culture Collection, Rochville, MD) were maintained in DMEM supplemented with 10\% FBS (HyClone Laboratories Inc., Logan, UT) and $100 \mathrm{U} / \mathrm{ml}$ of penicillin $\mathrm{G}$ plus $100 \mu \mathrm{g} / \mathrm{ml}$ of streptomycin (HyClone Laboratories Inc.).

\section{Cloning of KM-HN-1 and construction of expression vectors}

$\mathrm{KM}-\mathrm{HN}-1$ was initially obtained via yeast twohybrid screening using hCdc14A as bait. The obtained gene was a partial c-terminal fragment clone. In order to obtain the full length $\mathrm{KM}-\mathrm{HN}-1$, we employed the PCR method. In addition to the $\mathrm{PCR}$ cloning of $\mathrm{KM}-\mathrm{HN}-1$ we purchased a fulllength of IMAGE clone and verified it by DNA sequencing. The ORF of KM-HN-1 was further subcloned into pGEX (Pharmacia, Piscataway, NJ), pClneo (Promega, Madison, WI), pEGFP (Clontech, Palo Alto, CA) and pShuttle-EGFP adenoviral shuttle vectors.

\section{Northern blot analysis}

Human multiple tissue Northern blots (hMTN) were purchased from Clontech. The blots were prehybridized with MyracleHyb (Stratagen, La Jolla, 
CA) hybridization solution at $68^{\circ} \mathrm{C}$ for $2 \mathrm{~h}$, and then hybridized with the same prehybridization solution containing ${ }^{32} \mathrm{P}$-labeled $\mathrm{KM}-\mathrm{HN}-1$ probe for $6 \mathrm{~h}$. The hybridized hMTN blots were washed in $2 \times$ SSC containing $0.5 \%$ SDS twice for $15 \mathrm{~min}$ each and with $0.5 \times$ SSC containing $0.5 \%$ SDS twice for $15 \mathrm{~min}$ each, then exposed to X-ray film (Kodak, Rochester, NY) for $24 \mathrm{~h}$ for autoradiography.

\section{RT-PCR analysis of KM-HN-1}

$0.5 \mathrm{~g}$ of colon cancer tissues and surrounding normal tissues from surgical resection samples were used for RNA purification. Total RNA purification was conducted out using Trizol reagents (Invitrogen, San Diego, CA). After the precipitation of RNA with isopropanol, the RNA was dissolved in DEPC- treated water. For cDNA synthesis, $1 \mu \mathrm{g}$ of total RNA was incubated in $20 \mu \mathrm{l}$ of reaction mixture containing $2 \mu \mathrm{M}$ oligo-dT primer (Bioneer, Daejeon, Korea), MMLV reverse transcriptase (Enzynomics, Korea), RNasin (Promega) and 0.2 $\mathrm{mM}$ NTP for $30 \mathrm{~min}$ at $42^{\circ} \mathrm{C}$. After cDNA synthesis, PCR was performed for 30 cycles for the quantitation of KM-HN-1 and for 25 cycles for human actin. The sequences of the primers used were as follows: KM-HN-1 (380 bp product): forward, 5'TACAAGAATCTACTGCCAGAC-3'; reverse, 5'-ATCTGTTTTGGTTTCCATTACCAT-3'. Human actin (160bp product): forward, 5'-ATCATGAAGTGTGACGTGGACAT-3'; reverse, 5'-AGGAGGAGCAATGATCTTGATCT-3'. The PCR-products were electrophoresed on $2.5 \%$ NuSieve 3:1 agarose (FMC).

\section{Generation of antisera and immunoprecipitation}

To generate recombinant $\mathrm{KM}-\mathrm{HN}-1$, the $\mathrm{N}$-terminal half fragment (1-417) and C-terminal half fragment (418-833) were PCR-amplified and subcloned into pGEX4T-1 vector. After confirmation via DNA sequencing the recombinant GST fusion proteins were purified and immunized to rabbits in order to raise anti-sera. To purify the anti-KM-HN-N antibody, the immunized sera were first precleared with GST-bound GST agarose. The precleared anti-KM-HN-N antisera were further purified using GST-KM-HN-N (1-417) immobilized with Affigel-10 (Bio-Rad Laboratories). For immunoprecipitation, cultured cells were lysed in Tris-buffered saline containing $0.5 \%$ NP-40 and $0.5 \%$ sodium deoxycholate. The cell lysates were centrifuged at $15,000 \times g$ for $15 \mathrm{~min}$ at $4^{\circ} \mathrm{C}$. The supernatant was precleared with normal rabbit IgG complexed with protein-A agarose (Santa Cruz). The anti $\mathrm{KM}-\mathrm{HN}-1$ antisera bound by protein-A agarose were directly used for the immunoprecipitation of
$\mathrm{KM}-\mathrm{HN}-1$.

\section{Plasmid construction and generation of GFP tagged KM-HN-1 adenovirus}

Recombinant adenoviruses were constructed according to the procedure described previously (He et al., 1998; Seong et al., 2002). In brief, coding areas of the full ORF (1-833, KM-HN-full), $\mathrm{N}$-terminal $2 / 3$ region (1-557, KM-HN-N1), Nterminal $1 / 3$ region (1-278, KM-HN-N2), C-terminal $2 / 3$ region $(278-833, \mathrm{KM}-\mathrm{HN}-\mathrm{C} 1)$, C-terminal $1 / 3$ region (557-833, $\mathrm{KM}-\mathrm{HN}-\mathrm{C} 2$ ) and internal $1 / 3$ region (278-557, KM-HN-M) were PCR-amplified with Turbo pfu (Stratagene) and subcloned into the pShuttle-EGFP adenoviral shuttle vector. DNA sequencing and immunoblotting with anti-GFP antibody confirmed the construction of recombinant $\mathrm{KM}-\mathrm{HN}-1$. The appropriate recombinants were then digested with Pme1 and cotransformed into BJ5183 E.coli strain with the pAdEasy adenoviral backbone vector. The obtained recombinant adenoviral plasmids were transfected to 293 cells to generate $\mathrm{KM}-\mathrm{HN}-1$ expressing adenovirus. The expression of recombinant GFP fused KM-HN-1 was confirmed by immunoblotting using anti-GFP monoclonal antibody (JL-8, Clonetech).

\section{Antibodies for indirect immunofluorescence staining}

For indirect immunofluorescence staining the following proteins were visualized with the indicated antibodies and dilutions: KM-HN-1, 1:200 diluted affinity-purified anti $\mathrm{KM}-\mathrm{HN}-1$ antibody; $\gamma$-tubulin, 1:1,000 diluted GTU-88 mouse monoclonal antibody (Sigma). Secondary antibodies for immunofluorescence staining were AlexFluoro 488-conjugated goat anti rabbit IgG (Molecular Probes), Texas red conjugated goat anti-mouse IgG (Jackson Immunological Laboratories).

\section{IF staining and confocal microscopy}

U-2 OS cells were grown on Poly D-lysine (Sigma P0899) coated glass coverslips. After washing with PBS, the cells were fixed with PBS containing $3.7 \%$ formaldehyde solution, then the cells were permeabilized with PBS containing $0.5 \%$ Triton $X-100$. After blocking with PBS containing 5\% BSA solution, the coverslips were incubated with KM$\mathrm{HN}-1$ antibody, anti $\gamma$-tubulin antibody for $2 \mathrm{~h}$. After washing of coverslips, fluorescence-conjugated secondary antibodies were treated for $2 \mathrm{~h}$. For nuclear visualization Heoschst 33452 (Sigma) dye was used after seconary antibody incubation. The 


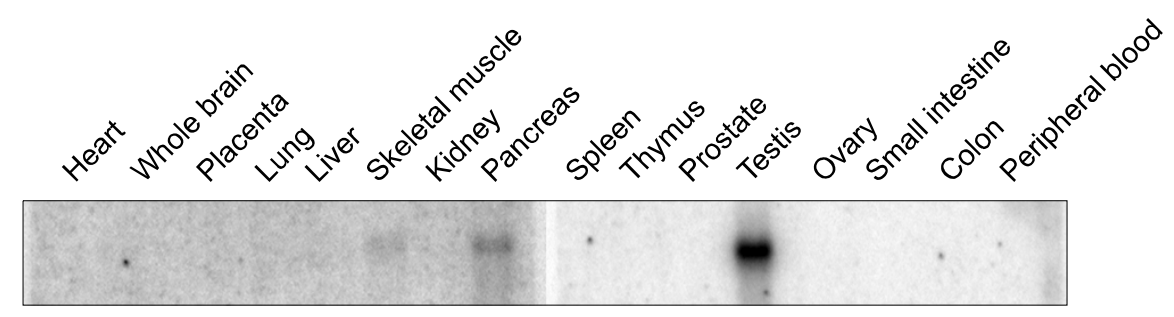

B

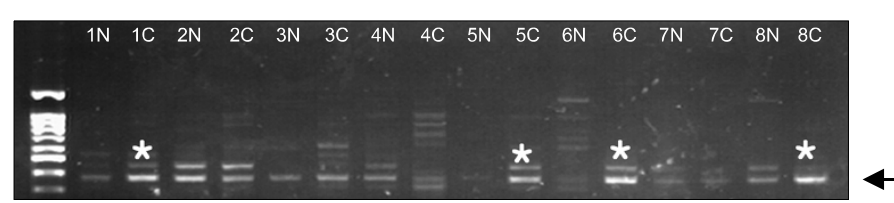

KM-HN-1 (380 bp)

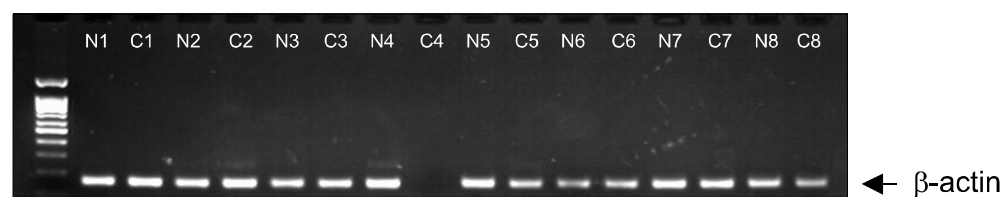

Figure 2. Northern blot and RT-PCR analysis of KM-HN-1. (A) Two kinds of humun MTN blots covering 16 different human tissues were used. The KM-HN-1 cDNA was labeled with $\mathrm{P}^{32}$ and probed. After hybridization, the membranes were washed and autoradiography was carried out. (B) RNAs of 8 samples of colon cancer tissues and surrounding normal tissues were used for RT-PCR analysis. The asterisk $\left({ }^{*}\right)$ represents $\mathrm{KM}-\mathrm{HN}-1$ messenger RNA increased lane. stained coverslips were mounted on glass slides with semi-solidifying mounting solution (Polyscience). Confocal fluorescence images were collected by Carl Zeiss LSM 510 Meta confocal microscope.

\section{Results}

\section{Expression of KM-HN-1 mRNA in normal tissues and colon cancers}

To detect the tissue-specific distribution pattern of $\mathrm{KM}-\mathrm{HN}-1$ in human tissue, human multiple tissue northern blots (hMTN) were used for northern hybridization analysis. This analysis detected a single band of approximately $4 \mathrm{kB}$ in the testis, pancreas and skeletal muscle. However, we failed to detect KM-HN-1 transcripts in the whole brain, heart, placenta, thymus, and ovary tissues. The expression of $\mathrm{KM}-\mathrm{HN}-1$ was exceptionally high in the testis (Figure 2A). The high expression levels of $\mathrm{KM}-\mathrm{HN}-1$ in the testis were consistent with previous Northern blot data from initial cloning report of KM-HN-1 (Monji et al., 2004). Due to the high proliferation activity in the testis, the high level of $\mathrm{KM}-\mathrm{HN}-1$ in testis suggests that it may play a crucial role in cell proliferation. We also analyzed the $\mathrm{KM}-\mathrm{HN}-1$ expression in several colon cancers. 4 out of 8 samples of colon cancer evidenced increased $\mathrm{KM}-\mathrm{HN}$-1 expression (Figure 2B).

\section{Production of antisera and immunoprecipitation of KM-HN-1}

In order to generate antisera against $\mathrm{KM}-\mathrm{HN}-1$, either the $\mathrm{N}$-terminal (1-417) or C-terminal domain (418-833) of KM-HN-1 ORF was cloned into pGEX-4T and the resultant fusion proteins were purified. Each GST-N-terminal KM-HN-1 and GSTC-terminal KM-HN-1 was used for production of antisera. The purified antisera detected the overexpressed $\mathrm{KM}-\mathrm{HN}-1$ and endogenous $\mathrm{KM}-\mathrm{HN}-1$ (Figure $3 \mathrm{~A}$ ). When competing with immunogen (GST-KM-HN-1 or C-terminal peptide) the binding of antisera to KM-HN-1 was effectively diminished (Data not shown). As is shown in Figure $3 \mathrm{~A}$, the antisera against the $\mathrm{N}$-terminal half of $\mathrm{KM}-\mathrm{HN}-1$ is capable of recognizing endogenous $\mathrm{KM}-\mathrm{HN}-1$ as a $105 \mathrm{kDa}$ protein. We also verified the existence of $\mathrm{KM}-\mathrm{HN}-1$ via immunoprecipitation with the C-terminal antibody followed by the immunoblotting of immnuoprecipitates using the $\mathrm{N}$-terminal antisera (Figure 3B).

\section{Colocalization of KM-HN-1 and hCdc14A}

hCdc14A is known to localize to the centrosome and to perform crucial functions in centrosome duplication and mitosis (Kaiser et al., 2002; Mailand et al., 2002; Trautmann and McCollum, 2002). As we obtained the $\mathrm{KM}-\mathrm{HN}-1$ gene via yeast two-hybrid screening using $\mathrm{hCdc} 14 \mathrm{~A}$ as bait, we tried to see the colocalization of $\mathrm{KM}-\mathrm{HN}-1$ with 
A

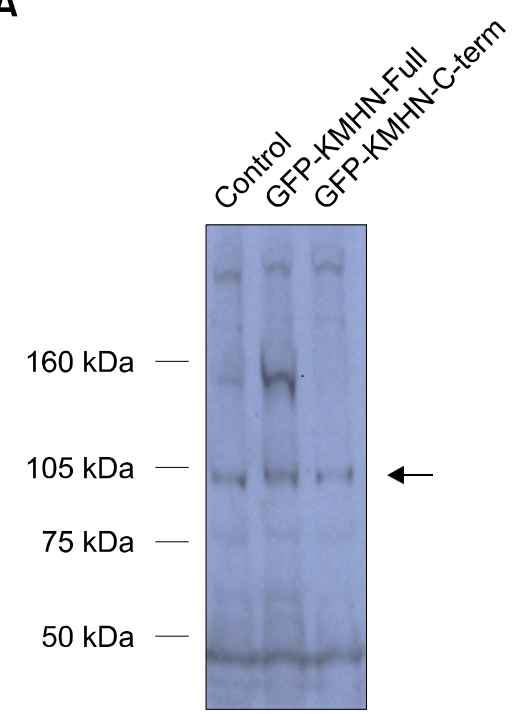

B

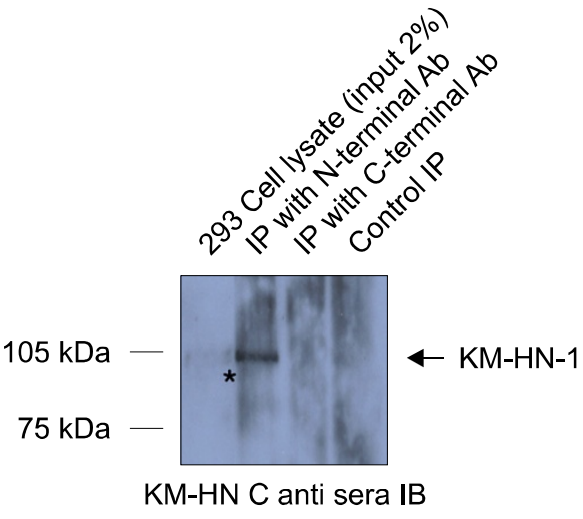

KM-HN N anti sera IB

C
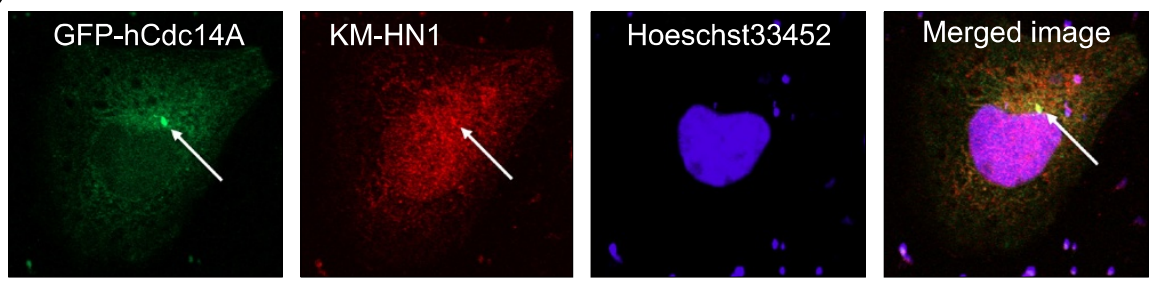

Figure 3. Production of KM-HN-1 antisera and identification of KM-HN-1. Two kinds of antisera were raised-one is against the N-terminal half of KM-HN-1 (anti-KM-HN-N antisera), and the other is against the C-terminal half of KM-HN-1 (anti-KM-HN-C antisera). (A) Immunoblotting using anti-KM-HN-N antisera, 293 cells were transfected with pShuttleCMV-GFP-KM-HN-full and pShuttleCMV-GFP-KM-HN-C. After $36 \mathrm{~h}$ of transfection, the cell lysates were electrophoresed and transferred to NC membranes, after which immunoblotting was carried out. Control means vector transfected cell. The arrowhead marks the GFP tagged full length of KM-HN-1, the arrow marks endogenous KM-HN-1. (B) Immunoblotting of immunoprecipitated KM-HN-1 protein. For immunoprecipitation 293 human embryonic kidney cells were lysed and was pulled down with indicated antisera. The immunoprecipitated samples were electrophoresed and Western blot analysis was carried out using anti-KM-HN-C antisera. The anti-KM-HN-C antisera cannot pull down endogenous KM-HN-1. The asterisk $\left(^{*}\right)$ represents two bands of endogenous KM-HN-1 (full length and splicing variant). (C) Colocalization of EGFP-hCdc14A with KM-HN-1. For colocalization analysis of KM-HN-1 and hCdc14A pEGFPC1-hCdc14A was transfected to U-2 OS cells. After $16 \mathrm{~h}$ of transfection, the cells were fixed in $3.7 \%$ formaldehyde then permeabilized with $0.5 \%$ Triton X-100 for $5 \mathrm{~min}$. For the staining of $\mathrm{KM}-\mathrm{HN}-1$ the rabbit polyclonal antiKM-HN-1 antisera was used. Green, EGFP-tagged full length of hCdc14A; red, KM-HN-1; Blue, Hoeschst 33452 dye for nuclear staining. Arrows mark the colocalization signal of KM-HN-1 and hCdc14A

hCdc14A. As is shown in Figure 3C, the EGFPTagged hCdc14A showed colocalization with KM$\mathrm{HN}-1$ in the centrosome area. However, we failed to observe apparent binding of $\mathrm{KM}-\mathrm{HN}-1$ with hCdc14A.

\section{Generations of GFP tagged KM-HN-1 deletion mutants}

The C-terminal region of $\mathrm{KM}-\mathrm{HN}-1$ harbored a coiled-coil region, which contains the SMC motif and shared a conserved region of homology with yeast spindle pole proteins Spc110p and Rad51p. In addition to the SMC motif, KM-HN-1 harbors a bipartite NLS motif and a leucine zipper motif in its internal $1 / 3$ fragment (Figure $1 A$ and $B$ ). The $\mathrm{KM}-\mathrm{HN}-1$ protein was shown to contain more than five leucine zipper repeats (Figure 1B). This region appeared to be important to proper cellular localization and function. To determine the function of each region in $\mathrm{KM}-\mathrm{HN}-1$, we expressed various forms of $\mathrm{KM}-\mathrm{HN}-1$ in U-2OS cells using adenoviral system (Figure 4A). These recombinant proteins were N-terminally fused with GFP to facilitate 
A

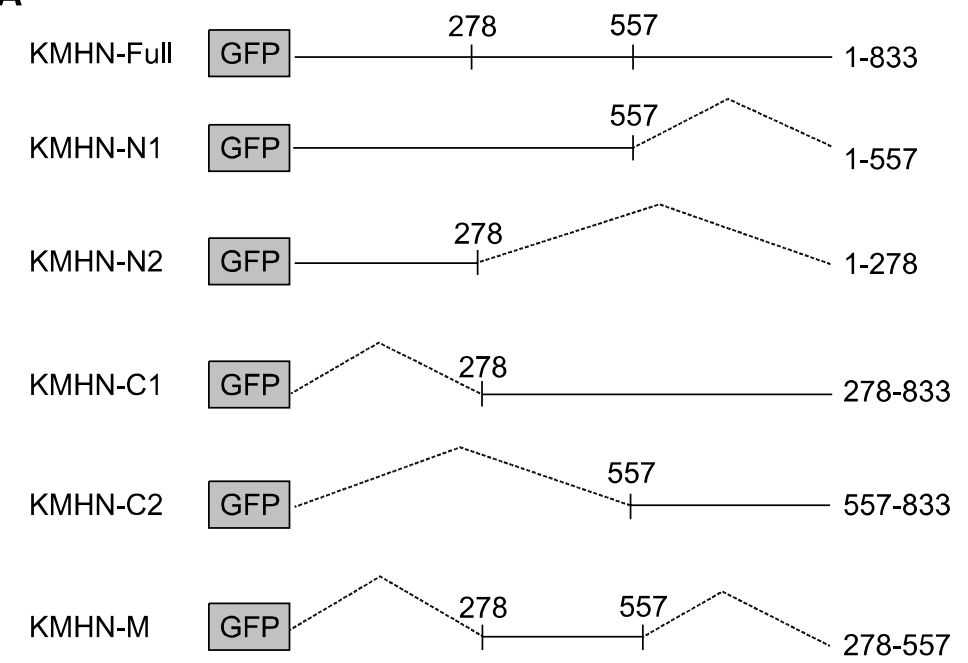

B

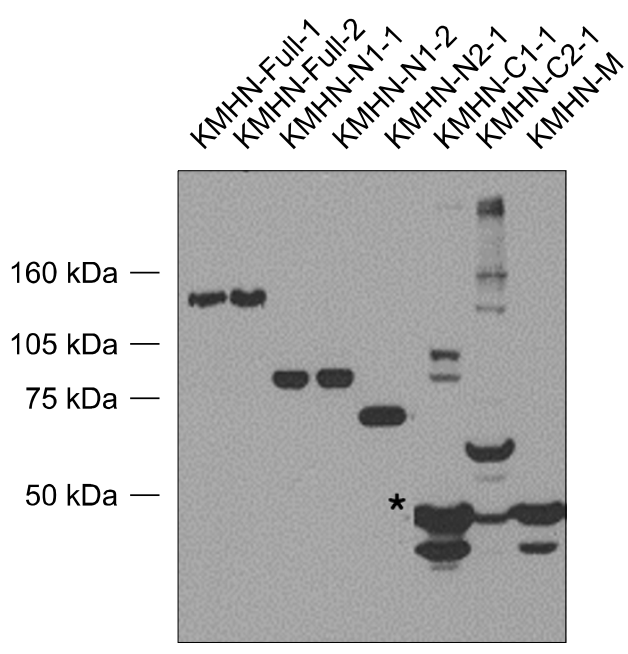

Figure 4. Generation of KM-HN-1 deletion mutant. (A) For the expression of five kinds of deletion mutants (N1, N2, C1, C2 and M) and full length of KM-HN-1 were fused with GFP and expressed via an adenoviral system. (B) Immunoblotting analysis of each virus infected cell. The recombinant adenovirus was infected into U-2 OS cell. The cell lysates were analyzed with Western blot analysis using GFP antibody (JL-8, Clonetech). In KM-HN-C1 immunoblotting the band marked with * is proteolytic cleavage fragment.

protein detection and subcellular localization. After $24 \mathrm{~h}$ of virus infection, the total cellular proteins were prepared for immunoblotting with anti-GFP antibody. As shown in Figure 4B, each GFP-fused proteins were expressed according to their assuming molecular weights. However in GFP-KM-HNC1 infected cell lysates the proteolytic cleavage products were dominant (marked * in Figure 4B) than the uncleaved product.

\section{Intracellular localization of endogenous KM-HN-1 and various deletion mutants}

To determine the intracellular localization of KM$\mathrm{HN}-1$ we carried out immunofluorescence staining using affinity-purified KM-HN-1 antibody. To validate the antibody specificity we also carried out immunofluorescence staining in the presence of competing immunogen (GST-KM-HN-1). As is shown in Figure 5, the endogenous $\mathrm{KM}-\mathrm{HN}-1$ (endo-KMHN in Figure 5) showed nuclear localization and colocalization with $\gamma$-tubulin, which make $\gamma$-TuRC and localize to the centrosomes. The competition of GST-KM-HN-1 with the endogenous $\mathrm{KM}-\mathrm{HN}-1$ effectively blocked the centrosomal signal of KM-HN-1 antibody (Competition). We also used C-terminal peptide (800-815) antibody for immunofluorescence staining. The employing of C-terminal peptide competition during immunofluorescence staining using C-terminal peptide antibody also blocked the centrosomal localization of $\mathrm{KM}-\mathrm{HN}-1$ (data not shown). The
GFP-tagged full length of $\mathrm{KM}-\mathrm{HN}-1$ also showed similar localization with endogenous $\mathrm{KM}-\mathrm{HN}-1$ protein (KMHN-full in Figure 5). The centrosomal localization signal of GFP-KM-HN-full was more prominent than that of the endogenous protein. The C-terminal 1/3 (KM-HN-C2) of KM-HN-1 also evidenced centrosomal localization, but the nuclear localization intensity was diminished (KMHN-C2 in Figure 5). Due to the abundance of the cleavage product, the GFP signal in GFP-KM-HN-C1 expressed cells was greatly superimposed with the cleavage product. As the anti-GFP antibody detects the cleavage product of GFP-KM-HN-C1 (Figure 4B) and the N-terminal Ser65-Tyr66-Gly67 of GFP is essential for proper fluorescence emission (Heim et al., 1994), the major cleavage product of GFP-KM-HN-C1 seems to be C-terminally cleaved and similar with GFP-KM-HN-M. Without the contaminating cleavage product of GFP-KM-HN-C1, the centrosomal localization signal of GFP-KM-HN-C1 might have been enhanced more. The N-terminal 2/3 of KM-HN-1 (KMHN-N1 in Figure 5) showed similar level of nuclear and cytoplasmic signal, the centrosomal signal of this protein was no less than that of KM-HN-C2. The $\mathrm{N}$-terminal $1 / 3$ of KM-HN-1 (KMHN-N2 in Figure 5) exhibited weakened centrosomal localization, but interestingly it showed strong nuclear localization. The internal $1 / 3$ fragment (KM-HN-M), which harbors putative NLS and leucine zipper motif, showed weakened centrosomal and nuclear localization (KMHN-M in Figure 5). The KM-HN-M over- 


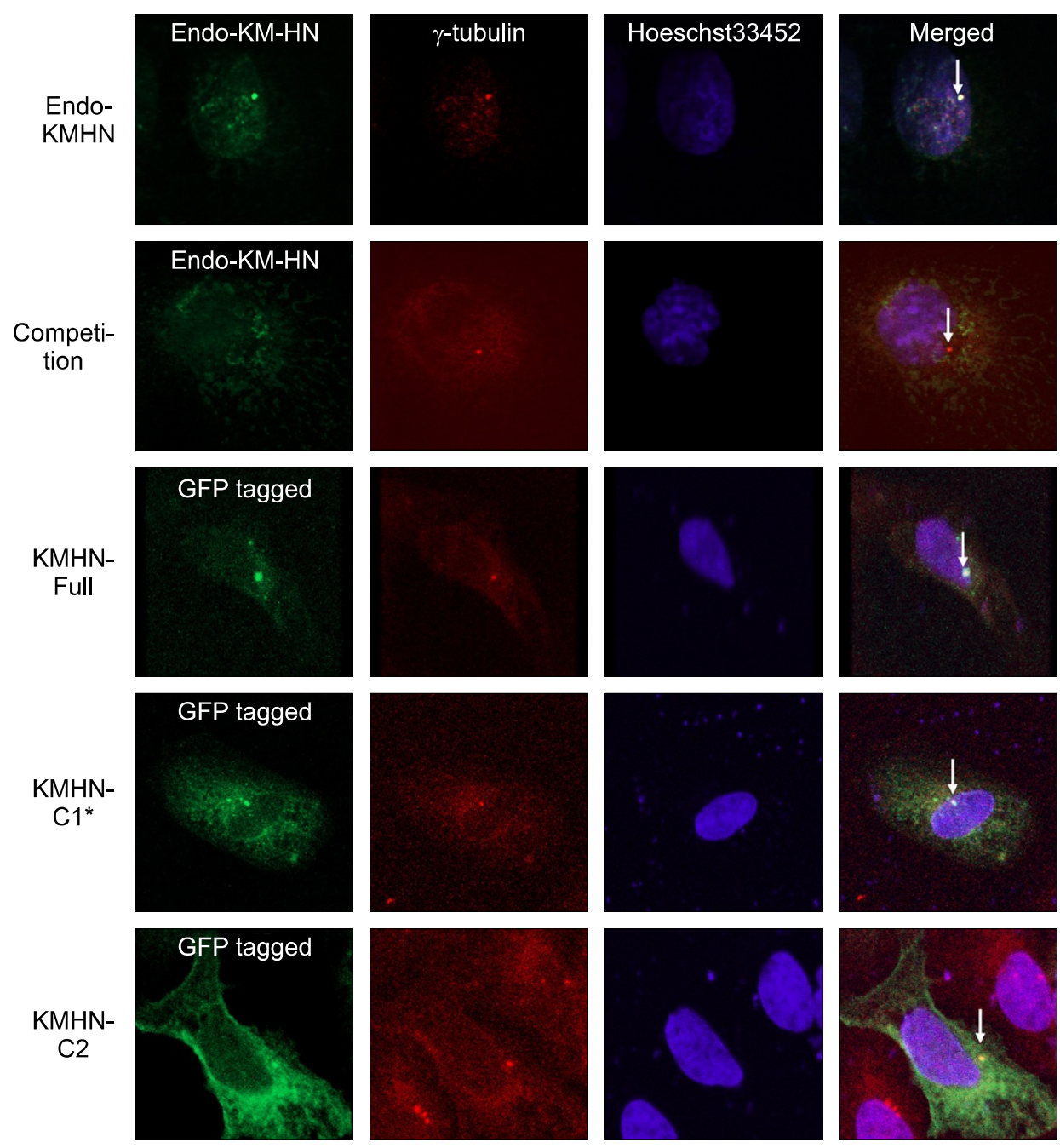

Figure 5. Intracellular localization of various kinds of $\mathrm{KM}-\mathrm{HN}-1$ deletion mutants and endogenous $\mathrm{KM}-\mathrm{HN}-1$. For expression of GFP-KM-HN-1 adenovirus was transduced into U-2 OS cells. After $16 \mathrm{~h}$ of infection cells were fixed in $3.7 \%$ formaldehyde then permeabilized with $0.5 \%$ Triton $\mathrm{X}-100$ for 5 min prior to the incubation of $\gamma$-tubulin antibodies. Endo-KMHN, endogenous KM-HN-1 protein probed with N-terminal affinity purified antisera; Competition, To see effective blocking of KM-HN-1 antibody purified GST-KM-HN-N was preincubated with KM-HN-1 antisera; Arrow marks the colocalization signal of $\mathrm{KM}-\mathrm{HN}-1$ and $\gamma$-tubulin. *As $\mathrm{KM}-\mathrm{HN}-\mathrm{C} 1$ contained cleaved product as majority, the localization profile cannot directly represent the localization of C-terminal 2/3 of KM-HN-1.

expressed cell evidenced a decreased $\gamma$-tubulin signal. The localization profiles of various $\mathrm{KM}-\mathrm{HN}-1$ constructs are summarized in Table 1. These results indicate that the cytoplasmic and centrosomal localization-determining region resides in the C-terminal region, and the nuclear localizationdetermining region is in the $\mathrm{N}$-terminal region.

\section{Forced overexpression of $\mathrm{KM}-\mathrm{HN}-1$ can generate aberrant $\gamma$-TuRC-like foci}

We attempted to determine whether the centrosomal localization of $\mathrm{KM}-\mathrm{HN}-1$ requires intact microtubule polymers. The nocodazole-treated cells also showed distinct localization to the centrosome (Figure 6), much like the untreated cells (endo$\mathrm{KMHN}$ in Figure 5). This result indicates that the centrosome localization is not dependent on microtubule integrity and dynamics. We also investigated whether overexpressed $\mathrm{KM}-\mathrm{HN}-1$ foci can recruit $\gamma$-TuRC. For prolonged overexpression we incubated for $36 \mathrm{~h}$ after infection of adv-GFP. $\mathrm{KM}-\mathrm{HN}$-full, then fixed and immunofluorescence staining was carried out. As is shown in Figure 7A and $B$, the ectopically overexpressed $\mathrm{KM}-\mathrm{HN}-1$ foci could induce $\gamma$-TuRC-like foci. Unlike endogenous $\mathrm{KM}-\mathrm{HN}-1$, superfluously overexpressed GFP tagged $\mathrm{KM}-\mathrm{HN}-1$ showed punctuate pattern (Figure 

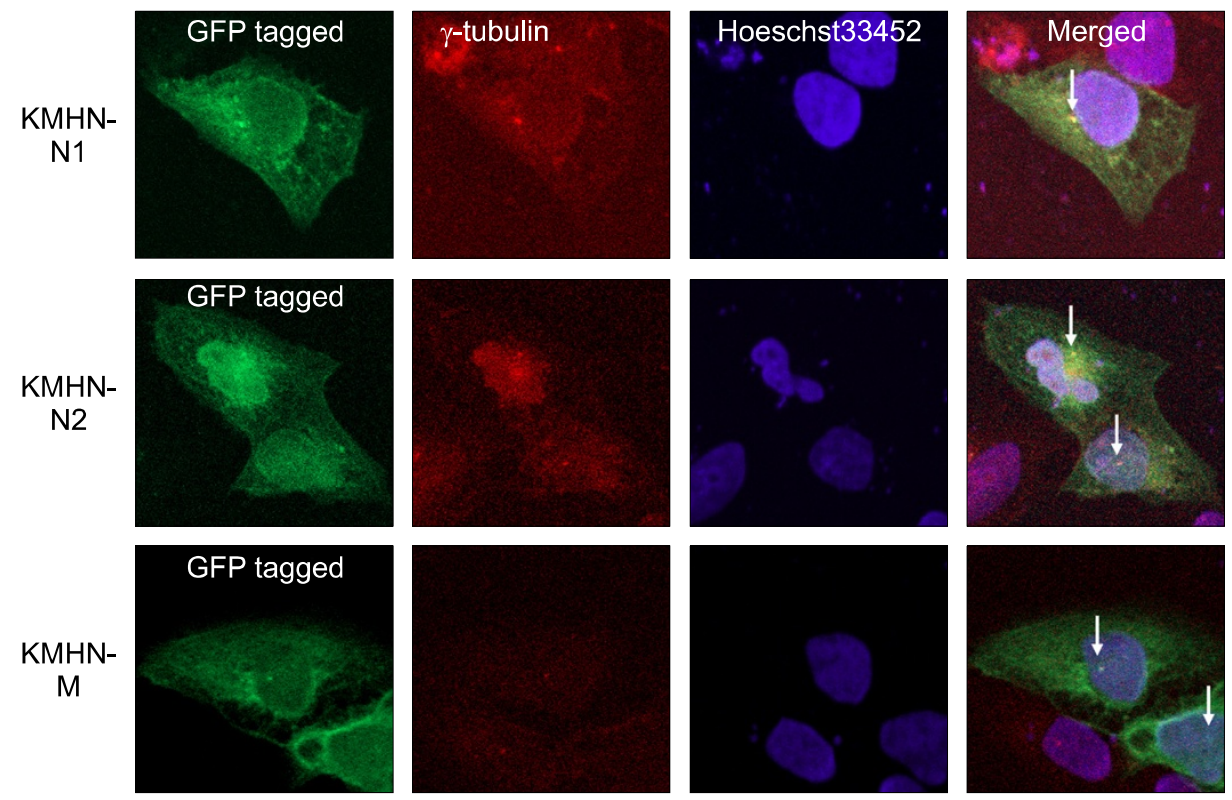

Figure 5. Continued.

Table 1. Summary of various GFP-KM-HN-1 protein localizations.

\begin{tabular}{lccc}
\hline & Centrosome & Cytoplasm & Nucleus \\
\hline KM-HN1 Full & +++ & + & + \\
KM-HN1 N1 & ++ & ++ & + \\
KM-HN1 N2 & +- & + & ++ \\
KM-HN1 C1* & +++ & ++ & - \\
KM-HN1 C2 & ++ & ++ & - \\
KM-HN1 M & + & ++ & +- \\
\hline
\end{tabular}

The relative intensity of centrosomal and nuclear localization of GFP-tagged KM-HN-1 mutants was measured and graded. ++++, most strong; + -, same as average of surrounding intracellular structures; -, not detectable. More than 15 cells were counted for each sample. *As $\mathrm{KM}-\mathrm{HN}-\mathrm{C} 1$ contained cleaved product as majority, the localization profile cannot wholly reflect the localization of C-terminal $2 / 3$ of $\mathrm{KM}-\mathrm{HN}-1$.

7A). This is due to the self-aggregation characteristics of overexpressed $\mathrm{KM}-\mathrm{HN}-1$ and does not represent the characteristics of intracellular localization pattern of endogenous $\mathrm{KM}-\mathrm{HN}-1$.

\section{Discussion}

Although $\mathrm{KM}-\mathrm{HN}-1$ has previously been identified as a cancer/testis antigen, the study of $\mathrm{KM}-\mathrm{HN}-1$ has mainly been limited to mRNA expression level analyses using several cancer tissues and cell lines (Monji et al., 2004; Condomines et al., 2007). $\mathrm{KM}-\mathrm{HN}-1$ has been shown to localize to the nu- cleus in interphase cells and chromosomes in mitotic cells, but the characterization of this protein at the protein level has not been addressed yet.

We isolated $\mathrm{KM}-\mathrm{HN}-1$ as an hCdc14A interacting protein from a yeast two-hybrid screening. We identified this protein as a $105 \mathrm{kDa}$ protein via immunoblotting and immunoprecipitation. The immunofluorescence analyses using GFP-KM-HN-1 and $\mathrm{N}$-terminal antisera revealed that $\mathrm{KM}-\mathrm{HN}-1$ mainly localize to centrosomes as well as nucleus. No chromosomal localization during mitosis was observed. Immunofluorescence staining using the C-terminal peptide antibody against the C-terminal 800-815 peptide residues of KM-HN-1 also showed similar centrosomal and nuclear localization (data not shown). Based on these results we concluded that the cancer/testis antigen KM-HN-1 is another member of centrosomal localizing proteins.

The centrosome is the principal microtubuleorganizing center of the cell and is surrounded by pericentriolar material, which tethers a variety of proteins, including $\gamma$-tubulin and hundreds of other proteins (Doxsey et al., 2005). The centrosome is crucial with regard to microtubule nucleation and anchorage, cell cycle regulation, cytokinesis, protein phosphorylation and signal transduction (Rieder et al., 2001; Doxsey et al., 2005). The hCdc14A localizes to the centrosomes and plays important role in centrosome cycle, chromosome segregation and anaphase progression (Bembenek and $\mathrm{Yu}$, 2001; Kaiser et al., 2002; Mailand et al., 2002). 

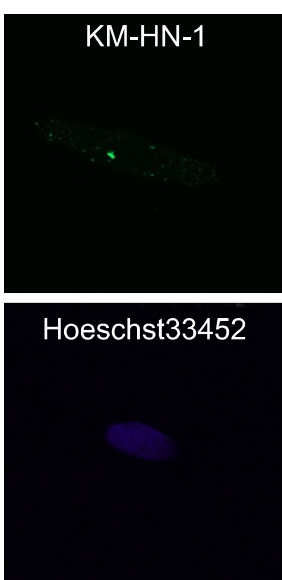

Endogenous KM-HN-1
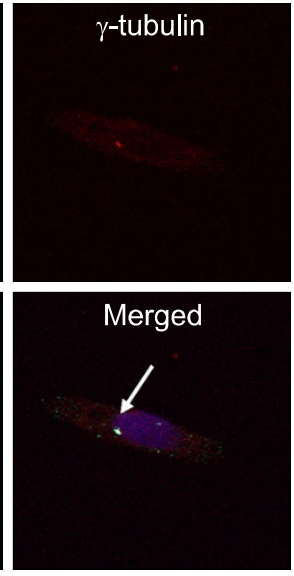
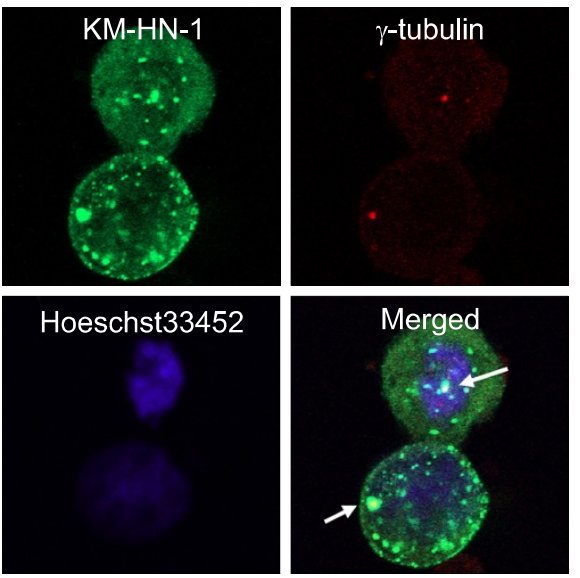

GFP-KM-HN-full

Figure 6. Microtubule depolymerization by nocodazole treatment does not alter centrosomal localization of $\mathrm{KM}-\mathrm{HN}-1$. For microtubule depolymerization, nocodazole U-2OS cells were treated at $200 \mathrm{nM}$ for $16 \mathrm{~h}$. For visualization of GFP-KM-HN-full under the microtubule depolymerized status the KM-HN-1 adenovirus and nocodazole was treated simultaneously to U-2OS cells. After $16 \mathrm{~h}$ of treatment the cells were fixed and permeabilized and immunofluorescence staining was performed. The arrows mark the centrosomal colocalization of $\mathrm{KM}-\mathrm{HN}-1$.

A
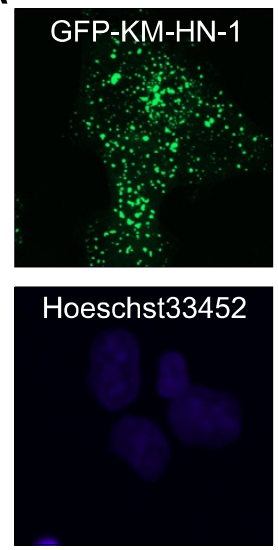
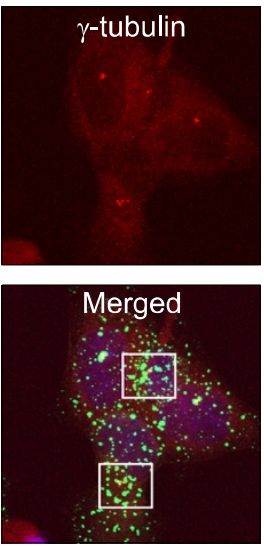

B
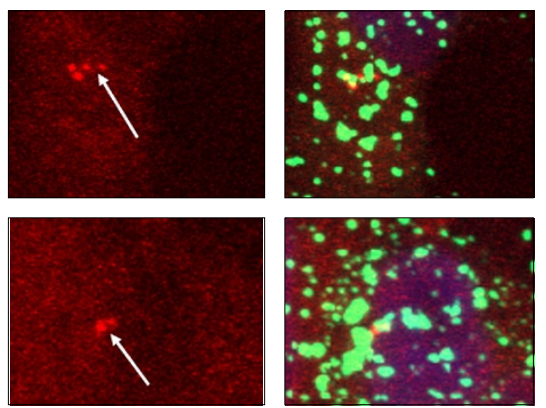

Figure 7. Forced overexpression of GFP-KM-HN-1 can induce aberrant $\gamma$-TuRC foci formation. (A) After $36 \mathrm{~h}$ of advGFP-KM-HN-1 infection, the cells were fixed and stained for $\gamma$-tubulin antibody. Green, overexpressed GFP tagged KM-HN-1; red, $\gamma$-tubulin; blue, Hoeschst 33452 dye for DNA staining. (B) The rectangular area of Figure 7 A was magnified for the visualization of $\gamma$-TuRC foci, and KM-HN-1. The arrow represents aberrant $\gamma$-TuRC foci.

The endogenous $\mathrm{KM}-\mathrm{HN}-1$ showed colocalization with GFP tagged hCdc14A (Figure 3C).

$\mathrm{KM}-\mathrm{HN}-1$ has three coiled-coil regions in the $\mathrm{C}$-terminal half of the protein. The coiled-coil region is comprised of more than two or three $\alpha$-helices wrapped around each other and forms helical structures (Lupas, 1996; Burkhard et al., 2001). The coiled-coil regions function as protein-protein interaction domains for further assembly of macromolecular structures. These coiled-coil region containing proteins play diverse biological functions as components of cytoskeleton, nuclear matrix, centrosomes, spindle pole bodies, centromeres, and Golgi apparatus (Rose and Meier, 2004). A lot of centrosome-localizing proteins have been shown to harbor coiled-coil motifs, and the integrity of these motifs appears to be important for centrosomal localization (Andersen et al., 2003; Rose and Meier, 2004). In addition to the coiled-coil motif in $\mathrm{C}$-terminal half region, the $\mathrm{KM}-\mathrm{HN}-1$ harbors leucine zipper and NLS motif in internal $1 / 3$ of this protein. To determine which region in $\mathrm{KM}-\mathrm{HN}-1$ is 
responsible for centrosomal and nuclear localizations, we generated various GFP-fused KM$\mathrm{HN}-1$ deletion mutants.

We observed that the GFP-KM-HN-1 C-terminal fragment which harbors coiled-coil region efficiently localized to the centrosomes, while the mutants lacking the coiled-coil region localized to centrosomes marginally. These results suggest that the centrosomal localization-determining region resides within the $\mathrm{C}$-terminal coiled-coil region. In addition to centrosomal localization, the GFP-KM$\mathrm{HN}-\mathrm{C} 2$ existed in cytoplasm. The cytoplasmic localization of this protein was due to the putative NES (Nuclear Export Signal) between amino acid residues 611-616. This region showed homology with conserved motif of NES (L-X-X-L-X-L, where L can be either L, I, V, F or M.) (la Cour et al., 2004). As well as centrosomes the endogenous $\mathrm{KM}-\mathrm{HN}-1$ also localized to the nucleus. The nuclear distribution of endogenous $\mathrm{KM}-\mathrm{HN}-1 \mathrm{~T}$ is concord with previous $\mathrm{KM}-\mathrm{HN}-1$ report (Monji et al., 2004). Among the various kinds of $\mathrm{KM}-\mathrm{HN}-1$ constructs, the N-terminal $1 / 3$ of it (KM-HN-N2) exhibited the most prominent nuclear localizations. However, the $\mathrm{N}$-terminal $1 / 3$ region of this protein harbors no distinct nuclear localization signal, suggesting that this region contains a yet unidentified NLS motif. Protein target site prediction using Internet WoLF PSORT (http://wolfpsort.org/) program (Horton et al., 2007) showed that the $\mathrm{N}$-terminal $1 / 3$ shared similar nuclear localization value with internal $1 / 3$ of $\mathrm{KM}-\mathrm{HN}-1$ even in the absence of putative NLS.

The KM-HN-1 harbors a leucine zipper motif in the internal $1 / 3$ region followed by coiled-coil region. For the dimerization of the leucine zippercontaining protein, the minimal number of leucine zipper repeats is four (Landschulz et al., 1988). In the case of KM-HN-1, the number of leucine zipper repeats is six. The leucine zipper motif has been known as protein dimerization region of various kinds of DNA binding proteins. Thus far, speriolin (Goto and Eddy, 2004), ninein (Bouckson-Castaing et al., 1996), Nek2 kinase (Fry et al., 1999) are known as leucine zipper-containing centrosomal proteins. The centrosomal localizing Nek2 kinase dimerizes via the leucine zipper region (Fry et al., 1999). The $\mathrm{N}$-terminal leucine zipper of speriolin is required for the targeting of this protein to centrosomes (Goto and Eddy, 2004). In ninein, one of the essential components of pericentriolar centrosomal material, the coiled-coil domain harboring three leucine zippers is essential for centrosomal localization (Stillwell et al., 2004). The leucine zipper containing KM-HN-N1 showed centrosomal localization despite the impaired C-terminal coiledcoil region. This may be due to the fact that the leucine zipper region can act as a dimerization domain with other leucine zipper containing centrosomal localizing proteins or $\mathrm{KM}-\mathrm{HN}-1$ itself. From these results, we could conclude that the leucine zipper motif also contributes the centrosomal localization of $\mathrm{KM}-\mathrm{HN}-1$. Although the leucine zipper fragment of $\mathrm{KM}-\mathrm{HN}-1$ with impaired coiled-coil region can lead this protein to centrosomes, the coiled-coil region seems to have more responsibility for centrosomal localization. The centrosomal signal of KM-HN-M, in which leucine zipper harbors but coiled-coil is disrupted was not so strong as C-terminal fragment of KM$\mathrm{HN}-1$ or full length of it. The coiled-coil fragment of $\mathrm{KM}-\mathrm{HN}-1$ lacking leucine zipper or leucine zipper motif without coiled-coil region can act as dominant negative or inhibitor of organization of centrosomes through binding and sequestration of other leucine zipper or coiled-coil motif containing centrosomal localization proteins.

The centrosomal localization of $\mathrm{KM}-\mathrm{HN}-1$ was not related with microtubule integrity or microtubule dynamics. Microtubule depolymerization by nocodazole treatment could not alter the centrosomal localization of $\mathrm{KM}-\mathrm{HN}-1$. The overexpression of ninein, a centrosome localizing leucine zipper and coiled-coil region containing protein, exhibited a mislocalization of $\gamma$-TuRC (Stillwell et al., 2004). The ectopic overexpression of GFP-KM-HN-full induced abnormal $\gamma$-TuRC foci. Forced overexpression of $\mathrm{KM}-\mathrm{HN}-1$ also resulted in multiply dotted signals among which the $\gamma$-TuRC signal appeared. The multiple dot signals are likely due to the inter-molecular interactions between $\mathrm{KM}-\mathrm{HN}-1$ proteins or other coiled-coil domain containing proteins.

In conclusion, the cancer/testis antigen $\mathrm{KM}-\mathrm{HN}-$ 1 is a new member of centrosome localizing proteins and the $\mathrm{C}$-terminal coiled-coil region and the leucine zipper motif are important for proper centrosomal localization. Further studies on the protein interaction and the function of $\mathrm{KM}-\mathrm{HN}-1$ at the centrosomes may provide new insights into the organizations and functions of centrosomes.

\section{Acknowledgements}

This work was supported by a Korean Research Foundation Grant funded by the Korean Government (KRF-0412004-E0041) and the National R \& D Program for Cancer Control, Ministry of Health and Welfare (Grant No. 0620170-1) to Seong YS (Dankook University, College of Medicine). We are grateful to Bae JS and Kwon OK for initial work on KM-HN-1. We also thanks to Lee KS for communication of results. 


\section{References}

Andersen JS, Wilkinson CJ, Mayor T, Mortensen P, Nigg EA, Mann M. Proteomic characterization of the human centrosome by protein correlation profiling. Nature 2003;426:570-4

Bembenek J, Yu H. Regulation of the anaphase-promoting complex by the dual specificity phosphatase human Cdc14a. J Biol Chem 2001;276:48237-42

Bouckson-Castaing V, Moudjou M, Ferguson DJ, Mucklow S, Belkaid Y, Milon G, Crocker PR. Molecular characterisation of ninein, a new coiled-coil protein of the centrosome. J Cell Sci 1996;109:179-90

Boulikas T. Nuclear localization signals (NLS). Crit Rev Eukaryot Gene Expr 1993;3:193-227

Burkhard P, Stetefeld J, Strelkov SV. Coiled coils: a highly versatile protein folding motif. Trends Cell Biol 2001;11:82-8

Cobbe N, Heck MM. SMCs in the world of chromosome biologyfrom prokaryotes to higher eukaryotes. J. Struct Biol 2000;129:123-43

Condomines M, Hose D, Raynaud P, Hundemer M, De Vos J, Baudard M, Moehler T, Pantesco V, Moos M, Schved JF, Rossi JF, Reme T, Goldschmidt H, Klein B. Cancer/testis genes in multiple myeloma: expression patterns and prognosis value determined by microarray analysis. J Immunol 2007;78:3307-15

Doxsey S, McCollum D, Theurkauf W. Centrosomes in cellular regulation. Annu Rev Cell Dev Biol 2005;21:411-34

Fry AM, Arnaud L, Nigg EA. Activity of the human centrosomal kinase, Nek2, depends on an unusual leucine zipper dimerization motif. J Biol Chem 1999;274:16304-10

Goto M, Eddy EM. Speriolin is a novel spermatogenic cell-specific centrosomal protein associated with the seventh WD motif of Cdc20. J Biol Chem 2004;279:42128-38

He TC, Zhou S, da Costa LT, Yu J, Kinzler KW, Vogelstein B. A simplified system for generating recombinant adenoviruses. Proc Natl Acad Sci USA 1998;95:2509-14

Heim R, Prasher DC, Tsien RY. Wavelength mutations and post translational autoxidation of green fluorescent protein. Proc Natl Acad Sci 1994;91:12501-4

Horton P, Park KJ, Obayashi T, Fujita N, Harada H, Adams-Collier CJ, Nakai K. WoLF PSORT: protein localization predictor. Nucleic Acids Res 2007;35(Web Server issue):W585-7
Kaiser BK, Zimmerman ZA, Charbonneau H, Jackson PK. Disruption of centrosome structure, chromosome segregation, and cytokinesis by misexpression of human Cdc14A phosphatase. Mol Biol Cell 2002;13:2289-2300

la Cour T, Kiemer L, Molgaard A, Gupta R, Skriver K, Brunak $\mathrm{S}$. Analysis and prediction of leucine-rich nuclear export signals. Protein Eng Des Sel 2004;17:527-36

Landschulz WH, Johnson PF, McKnight SL. The leucine zipper: a hypothetical structure common to a new class of DNA binding proteins. Science 1988;240:1759-64

Lupas A. Coiled coils: new structures and new functions. Trends Biochem Sci 1996;21:375-82

Mailand N, Lukas C, Kaiser BK, Jackson PK, Bartek J, Lukas J. Deregulated human Cdc14A phosphatase disrupts centrosome separation and chromosome segregation. Nat Cell Biol 2002;4:317-22

Monji M, Nakatsura T, Senju S, Yoshitake Y, Sawatsubashi M, Shinohara M, Kageshita T, Ono T, Inokuchi A, Nishimura $\mathrm{Y}$. Identification of a novel human cancer/testis antigen, $\mathrm{KM}-\mathrm{HN}-1$, recognized by cellular and humoral immune responses. Clin Cancer Res 2004;10:6047-57

Pereira G, Schiebel E. The role of the yeast spindle pole body and the mammalian centrosome in regulating late mitotic events. Curr Opin Cell Biol 2001;13:762-9

Rieder CL, Faruki S, Khodjakov A. The centrosome in vertebrates: more than a microtubule-organizing center. Trends Cell Biol 2001;11:413-9

Rose A, Meier I. Scaffolds, levers, rods and springs: diverse cellular functions of long coiled-coil proteins. Cell Mol Life Sci 2004;61:1996-2009

Seong YS, Kamijo K, Lee JS, Fernandez E, Kuriyama R, Miki $\mathrm{T}$, Lee KS. A spindle checkpoint arrest and a cytokinesis failure by the dominant-negative polo-box domain of Plk1 in U-2 OS cells. J Biol Chem 2002;277:32282-93

Stegmeier F, Amon A. Closing mitosis: the functions of the Cdc14 phosphatase and its regulation. Annu Rev Genet 2004;38:203-32

Stillwell EE, Zhou J, Joshi HC. Human ninein is a centrosomal autoantigen recognized by CREST patient sera and plays a regulatory role in microtubule nucleation. Cell Cycle 2004;3: 923-30

Trautmann S, McCollum D. Cell cycle: new functions for Cdc14 family phosphatases. Curr Biol 2002;12:R733-5 